Research Paper

\title{
Statins Dose-Dependently Exert Significant Chemopreventive Effects Against Various Cancers in Chronic Obstructive Pulmonary Disease Patients: A Population-Based Cohort Study
}

\author{
Chun-Chao Chen ${ }^{1 *}$, Yi-Ping Hsu1 ${ }^{*}$, Ju-Chi Liu ${ }^{1,4^{*}}$, Pai-Feng Kao1,4, Li-Chin Sung1,4, Chao-Feng Lin ${ }^{1}$, Wen-Rui \\ $\mathrm{Hao}^{1}$, Shing-Hwa Liu², Szu-Yuan $\mathrm{Wu}^{2,3,4,5 \bowtie}$ \\ 1. Division of Cardiovascular Medicine, Department of Internal Medicine, Shuang Ho Hospital, Taipei Medical University, New Taipei City, Taiwan \\ 2. Institute of Toxicology, College of Medicine, National Taiwan University, Taipei, Taiwan \\ 3. Department of Radiation Oncology, Wan Fang Hospital, Taipei Medical University, Taipei, Taiwan \\ 4. Department of Internal Medicine, School of Medicine, College of Medicine, Taipei Medical University, Taipei, Taiwan \\ 5. Department of Biotechnology, Hungkuang University, Taichung, Taiwan. \\ ${ }^{*}$ These authors contributed equally to this study (joint first authors). \\ $\square$ Corresponding author: Szu-Yuan Wu, M.D., M.P.H. Attending Physician, Department of Radiation Oncology, Taipei Medical University and Wan Fang \\ Medical Center, No. 111, Section 3, Hsing-Long Road, Taipei 116, Taiwan, R.O.C E-mail: szuyuanwu4399@gmail.com.
}

( ) Ivyspring International Publisher. Reproduction is permitted for personal, noncommercial use, provided that the article is in whole, unmodified, and properly cited. See http://ivyspring.com/terms for terms and conditions.

Received: 2016.04.08; Accepted: 2016.06.29; Published: 2016.09.13

\begin{abstract}
PURPOSE: Chronic obstructive pulmonary disease (COPD) is associated with an increased cancer risk. We evaluated the chemopreventive effect of statins against all cancers in COPD patients and identified the statin with the strongest chemopreventive effect.

PATIENTS AND METHODS: All patients diagnosed with COPD at health care facilities in Taiwan $(n=116,017)$ from January 1, 2001, to December 31, 2012, were recruited. Each patient was followed to assess the following protective and risk factors for all cancers: age; sex; comorbidities (diabetes, hypertension, dyslipidemia) and the Charlson comorbidity index $[\mathrm{CCl}]$ ); urbanization level; monthly income; and nonstatin drug use. The index date of statins use was the date of COPD confirmation. Propensity scores (PSs) were derived using a logistic regression model to estimate the effect of statins by considering the covariates predicting intervention (statins) receipt. To examine the dose-response relationship, we categorized statin use into four groups in each cohort (<28 [statin nonusers], 28-90, 91-365, and >365 cumulative defined daily dose).

RESULTS: After PS adjustment for age, sex, CCl, diabetes, hypertension, dyslipidemia, urbanization level, and monthly income, we analyzed the all-cancer risk. The adjusted hazard ratios (aHRs) for the all-cancer risk were lower among statin users than among statin nonusers (aHR = $0.46,95 \%$ confidence interval: 0.43 to 0.50 ). The aHRs for the all-cancer risk were lower among patients using rosuvastatin, simvastatin, atorvastatin, pravastatin, and fluvastatin than among statin nonusers (aHRs $=0.42,0.55,0.59,0.66$, and 0.78 , respectively). Sensitivity analysis indicated that statins dose-dependently reduced the all-cancer risk.

CONCLUSION: Statins dose-dependently exert a significant chemopreventive effect against various cancers in COPD patients. In particular, rosuvastatin has the strongest chemopreventive effect.
\end{abstract}

Key words: statin; COPD; cancer. 


\section{Introduction}

Chronic obstructive pulmonary disease (COPD) is a common respiratory condition characterized by airflow limitation [1] and airway and systemic inflammation. [2] Inflammation alone or combined with other factors influences cancer risk in humans [3]; the most common link between inflammation and cancer risk in COPD patients is aberrant inflammation and immunity. [4] COPD is primarily caused by smoking. However, COPD has been independently associated with an increased risk of lung cancer and is probably associated with the inflammation and scarring accompanying COPD development. [5, 6] In COPD patients, oxidized low-density lipoprotein (LDL) levels are high and are associated with lung function, inflammation, and oxidative stress. [7]

Lipid metabolism disorders are risk factors for several cancers.[8-10] In COPD patients, a common potential mechanism by which major risk factors such as smoking, hyperlipidemia, obesity, and hypertension lead to chronic diseases is systemic inflammation.[11, 12] A meta-analysis of 28 case-control studies and 17 observational cohort studies revealed an increased lung cancer risk associated with an affected relative risk of 1.8.[13] Taken together, COPD patients are at a high risk of cancers throughout their lives.

Statin therapy has various effects that may contribute to reducing the cancer risk, such as anti-inflammatory, antioxidant, and antiplatelet effects as well as lipid modification.[14-17] Reduced monocyte adhesion to the endothelium, reduced oxidative stress modification of LDLs, and increased mobilization and differentiation of endothelial progenitor cells also are potential benefits of lipid-lowering therapy.[18, 19] Thus, statins can be chemopreventive agents for COPD patients with chronic systemic inflammation and hyperlipidemia. Some meta-analyses of randomized trials have consistently revealed that statins do not affect cancer incidence and cancer mortality; however, this may be because of the selection of study populations that are not at a high cancer risk. [20,21]

In this study, we evaluated the chemopreventive effects of statins against all cancers in COPD patients, who are at a high risk of cancer because of chronic systemic inflammation, hyperlipidemia, and higher oxidative stress. In addition, we investigated the dose-dependence of this chemopreventive effect and evaluated the potential of anticancer effects of different types of statins.

\section{Patients and Methods}

The National Health Insurance (NHI) program, established in 1995, currently provides comprehensive health insurance coverage to $98 \%$ of the more than 23 million people in Taiwan. We used data from the NHI Research Database (NHIRD). Distributions of age, sex, and health care costs in the NHIRD and among NHI enrollees do not differ significantly. Data that can be used to identify patients or care providers, including the names of medical institutions and physicians, are encrypted before being sent to the National Health Research Institutes for inclusion in the NHIRD. The institutes further encrypt the data before releasing the database to researchers. Theoretically, the NHIRD data alone is insufficient to identify any individual. All researchers using the NHIRD and its data subsets must sign a written agreement declaring that they have no intention of attempting to obtain information that could potentially violate the privacy of patients or care providers. [22]

Our study cohort comprised all patients diagnosed with COPD (according to International Classification of Diseases, Ninth Revision, Clinical Modification [ICD-9-CM] codes) at health care facilities in Taiwan $(\mathrm{n}=116,017)$ between January 1 , 2001, and December 31, 2012. We excluded patients without a subsequent outpatient visit, emergency department visit, or inpatient hospitalization for COPD within 12 months of the first presentation ( $\mathrm{n}=$ $48,212)$; these patients were considered to not have COPD (Fig 1). We also excluded 15,436 patients aged younger than 40 years $(n=52,369)$ and had any cancer-related inpatient or outpatient diagnoses before the index date $(\mathrm{n}=5,353)$ or had been prescribed any statins within 6 months before the index date $(\mathrm{n}=3,214)$.

Our final study cohort contained 43,802 patients diagnosed with COPD in Taiwan over the 11-year period; of these, 10,086 used statins and 33,716 did not (Table 1). Each patient was followed to assess the risk and protective factors for all cancers. In addition, we considered factors such as demographic characteristics (age and sex); comorbidities (diabetes, hypertension, dyslipidemia) and the Charlson comorbidity index (CCI); urbanization level; monthly income; and the use of nonstatin lipid-lowering drugs, metformin, aspirin, and angiotensin-converting enzyme inhibitor (ACEI). The index date of statin use was the date of COPD confirmation. Because we aimed to evaluate the preventive effects of statin use in COPD patients having a high all-cancer risk, the primary endpoint was the all-cancer risk and the secondary endpoints were the differential benefits of various doses and types of statins. The defined daily dose (DDD)-recommended by the World Health Organization-is a measure of the prescribed drug 
amount. DDD is the assumed average maintenance dose per day of a drug consumed for its main indication in adults. [23] To examine the dose-response relationship, we categorized statin use into four groups in each cohort $(<28,28-90,91-365$, and $>365$ cumulative DDDs [cDDDs]) because the duration of the refill card was 3 months. Patients receiving $<28$ cDDDs were defined as statin nonusers (Tables 2-4). [24] Furthermore, to examine the preventive effect of different types of statins, we categorized statin use into different individual statin use groups in each cohort (Table 3).
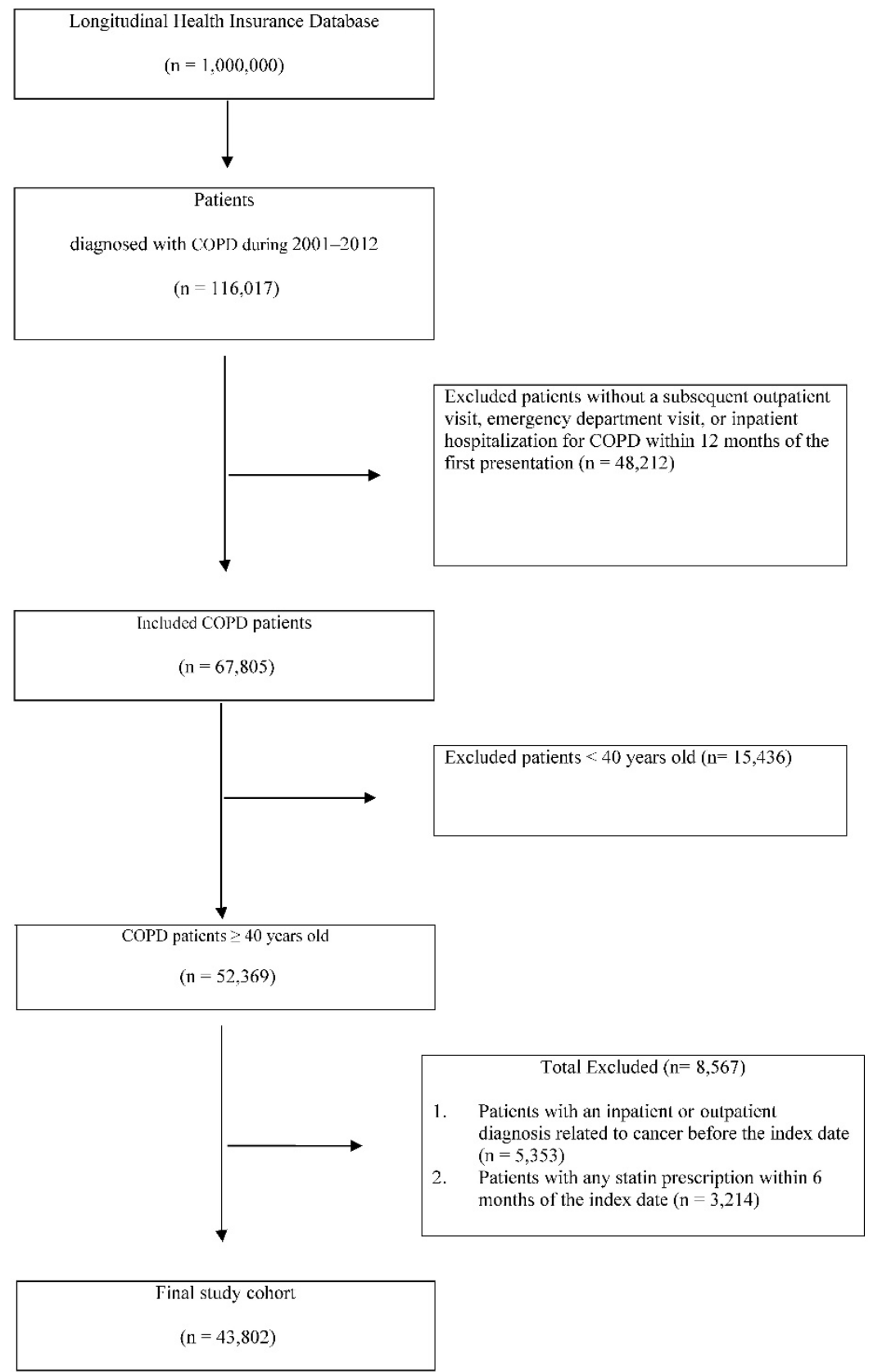

Figure 1. Patient selection flowchart.
Propensity scores (PSs) were derived using a logistic regression model to estimate the effect of statins by accounting for the covariates predicting receiving the intervention (statins). This method is commonly used in observational studies to reduce selection bias. [25] The covariates in the main model were PS adjusted for age, sex, CCI, diabetes, hypertension, dyslipidemia, urbanization level, and monthly income in New Taiwan dollars (NT\$0, NT\$1-21,000, NT\$21,000-33,300; and $\geq \mathrm{NT} \$ 33,301$; Table 2). The endpoint for both statin users and nonusers was the diagnosis of all cancers (ICD-9-CM 140-209) with a subsequent outpatient visit, emergency department visit, or inpatient hospitalization for any cancer within 12 months of diagnosis; the nonusers were used as the reference arm. The cumulative incidence of any cancer in the two groups was estimated using the Kaplan-Meier method.

A time-dependent Cox proportional hazard model was used to calculate the hazard ratios (HRs) of the all-cancer risk in the statin users and nonusers. The HRs were adjusted for age, sex, CCI, diabetes, hypertension, dyslipidemia, urbanization level, and monthly income in the multivariate analysis. A stratified analysis was conducted to evaluate the effect of statin use on age and sex (Table 2). All analyses were conducted using SAS software (Version 9.3; SAS, Cary, NC, USA); two-tailed $P<0.05$ was considered significant. In sensitivity analyses, external adjustments are used to improve the understanding of the effects of drugs and other covariates in epidemiological database studies. [26] Hence, in our sensitivity analyses, data were adjusted in different models to estimate the association of all-cancer incidence with age, sex, diabetes, dyslipidemia, hypertension, CCI, anxiety disorder, and the use of nonstatin lipid-lowering drugs, metformin, aspirin, and ACEI. The drug use-stratified models were adjusted for covariates in the main model and for each additional covariate (Table 4).

\section{Results}

Our COPD cohort comprised 43,802 patients; of these, 10,086 (30\%) used statins and the remaining 33,716 
(70\%) did not (Table 1). The total follow-up duration was 194,933.6 and 80,239.4 person-years for the statin nonusers and users, respectively. Compared with the statin nonusers, the statin users exhibited a higher prevalence of pre-existing medical comorbidities, such as diabetes, hypertension, and dyslipidemia, and a higher CCI (all $P<0.001$ ). In addition, significant differences were observed between the two groups in the distributions of age, sex, monthly income, and urbanization level as well as the use of nonstatin lipid-lowering drug, aspirin, ACEI, and metformin (Table 1). A higher proportion of statin nonusers used nonstatin lipid-lowering drugs, metformin, ACEI, and aspirin for <28 days; however, most statin users used these drugs for $>365$ days. A lower proportion of statin nonusers had a monthly income of $\geq \mathrm{NT} \$ 33,301$ or resided in urban areas. Table 2 shows the all-cancer risk among the statin nonusers and users. After PS adjustment for age, sex, CCI, diabetes, hypertension, dyslipidemia, urbanization level, and monthly income, we analyzed the all-cancer risk. The adjusted HRs (aHRs) for the all-cancer risk were lower among the statin users than among the statin nonusers (aHR $=0.46,95 \%$ confidence interval $[\mathrm{CI}]: 0.43$ to 0.50 ). The stratified analysis showed that the aHRs were significantly lower in the statin users, particularly those aged 40-74 years, regardless of sex. Specifically, the aHRs for the all-cancer risk were lower in the statin users than in the statin nonusers for every age group (40-64, 65-74, and $\geq 75$ years; aHRs $=0.43,0.45$, and 0.51 , respectively). The statin users also exhibited lower aHRs for the all-cancer risk than the statin nonusers did, after sex stratification (women: $\mathrm{aHR}=$ $0.44,95 \%$ CI: 0.40 to 0.50 ; men: $\mathrm{aHR}=0.48,95 \% \mathrm{CI}$ : 0.43 to 0.52 ).

Statins dose-dependently reduced the all-cancer risk in different cDDD subgroups; the main model was PS adjusted for age, sex, CCI, diabetes, hypertension, dyslipidemia, urbanization level, and monthly income (Table 3). Lipophilia statins comprised simvastatin, lovastatin, atorvastatin, and fluvastatin, whereas hydrophilia statins comprised pravastatin and rosuvastatin. Table 3 presents the all-cancer risk reduction demonstrated by lipophilia and hydrophilia statins in patients with COPD along with the doses and responses $(P$ for trend $<0.001)$. Among individual statins, lovastatin did not reduce the all-cancer risk in patients with COPD significantly. The aHRs for the all-cancer risk in patients using rosuvastatin, simvastatin, atorvastatin, pravastatin, and fluvastatin were lower than those of statin nonusers (aHRs $=0.42,0.55,0.59,0.66$ and 0.78 , respectively). Our results revealed that individual statins reduced the all-cancer risk at varying efficacies among COPD patients.

In the sensitivity analysis, PS adjustments were made to estimate the associations of age, sex, CCI, diabetes, hypertension, dyslipidemia, urbanization level, monthly income, and nonstatin lipid-lowering drugs, metformin, ACEI, and aspirin use with the incidence of all cancers in different models. Table 4 shows that the effects of statins remained significant in the subgroups of various covariates when the main model was adjusted for PSs. Statins dose-dependently reduced the all-cancer risk in all subgroups and the main model with additional covariates (nonstatin lipid-lowering drugs, metformin, ACEI, or aspirin use). All aHRs indicated that statins dose-dependently induced significant reductions in the all-cancer risk in all subgroups, regardless of comorbidities or drug use $(P<0.001)$. Thus, our data revealed that statins show a dose-dependent chemopreventive effect against all cancers.

Table 1. Characteristics of the Sample Population.

\begin{tabular}{|c|c|c|c|c|c|c|c|}
\hline & \multicolumn{2}{|c|}{$\begin{array}{l}\text { Entire cohort } \\
(\mathrm{n}=43,802)\end{array}$} & \multicolumn{2}{|c|}{$\begin{array}{l}\text { Patients using statins }(\geq 28 \\
\text { cDDDs; } \mathrm{n}=10,086)\end{array}$} & \multicolumn{2}{|c|}{$\begin{array}{l}\text { Patients not using statins }(<28 \\
\text { cDDDs; } \mathrm{n}=33,716)\end{array}$} & \multirow[t]{2}{*}{$P$ a } \\
\hline & $\mathrm{n}$ & $\%$ & $\mathrm{n}$ & $\%$ & $\mathrm{n}$ & $\%$ & \\
\hline Age, years (mean $\pm S D$ ) & \multicolumn{2}{|c|}{$62.92(13.18)$} & \multicolumn{2}{|c|}{61.55 (10.97) } & \multicolumn{2}{|c|}{$63.33(13.74)$} & $<0.001$ \\
\hline $40-54$ & 14458 & 33.01 & 3180 & 31.53 & 11278 & 33.45 & $<0.001$ \\
\hline $55-64$ & 9644 & 22.02 & 2899 & 28.74 & 6745 & 20.01 & \\
\hline $65-74$ & 10455 & 23.87 & 2777 & 27.53 & 7678 & 22.77 & \\
\hline$\geq 75$ & 9245 & 21.11 & 1230 & 12.20 & 8015 & 23.77 & \\
\hline \multicolumn{8}{|l|}{ Sex } \\
\hline Female & 19715 & 45.01 & 5150 & 51.06 & 14565 & 43.20 & $<0.001$ \\
\hline Male & 24087 & 54.99 & 4936 & 48.94 & 19151 & 56.80 & \\
\hline \multicolumn{8}{|l|}{$\mathrm{CCI}^{+}$} \\
\hline 0 & 11279 & 25.75 & 2586 & 25.64 & 8693 & 25.78 & $<0.001$ \\
\hline 1 & 12597 & 28.76 & 3014 & 29.88 & 9583 & 28.42 & \\
\hline 2 & 9075 & 20.72 & 2195 & 21.76 & 6880 & 20.41 & \\
\hline$\geq 3$ & 10851 & 24.77 & 2291 & 22.71 & 8560 & 25.39 & \\
\hline \multicolumn{8}{|l|}{ Diabetes } \\
\hline No & 33491 & 76.46 & 6819 & 67.61 & 26672 & 79.11 & $<0.001$ \\
\hline Yes & 10311 & 23.54 & 3267 & 32.39 & 7044 & 20.89 & \\
\hline
\end{tabular}




\begin{tabular}{|c|c|c|c|c|c|c|c|}
\hline & \multicolumn{2}{|c|}{$\begin{array}{l}\text { Entire cohort } \\
(\mathrm{n}=43,802)\end{array}$} & \multicolumn{2}{|c|}{$\begin{array}{l}\text { Patients using statins }(\geq 28 \\
\text { cDDDs; } \mathrm{n}=10,086)\end{array}$} & \multicolumn{2}{|c|}{$\begin{array}{l}\text { Patients not using statins }(<28 \\
\text { cDDDs; } n=33,716)\end{array}$} & \multirow[t]{2}{*}{$P a$} \\
\hline & $\mathrm{n}$ & $\%$ & $\mathrm{n}$ & $\%$ & $\mathrm{n}$ & $\%$ & \\
\hline \multicolumn{8}{|l|}{ Hypertension } \\
\hline No & 22067 & 50.38 & 4158 & 41.23 & 17909 & 53.12 & $<0.001$ \\
\hline Yes & 21735 & 49.62 & 5928 & 58.77 & 15807 & 46.88 & \\
\hline \multicolumn{8}{|l|}{ Dyslipidemia } \\
\hline No & 31731 & 72.44 & 5785 & 57.36 & 25946 & 76.95 & $<0.001$ \\
\hline Yes & 12071 & 27.56 & 4301 & 42.64 & 7770 & 23.05 & \\
\hline \multicolumn{8}{|c|}{ Nonstatin lipid-lowering drugs } \\
\hline$<28$ days & 39267 & 89.65 & 7212 & 71.51 & 32055 & 95.07 & $<0.001$ \\
\hline 28-365 days & 3186 & 7.27 & 1923 & 19.07 & 1263 & 3.75 & \\
\hline$>365$ days & 1349 & 3.08 & 951 & 9.43 & 398 & 1.18 & \\
\hline \multicolumn{8}{|l|}{ Metformin } \\
\hline$<28$ days & 35961 & 82.10 & 6286 & 62.32 & 29675 & 88.01 & $<0.001$ \\
\hline 28-365 days & 2684 & 6.13 & 964 & 9.56 & 1720 & 5.10 & \\
\hline$>365$ days & 5157 & 11.77 & 2836 & 28.12 & 2321 & 6.88 & \\
\hline \multicolumn{8}{|l|}{ ACEI } \\
\hline$<28$ days & 23928 & 54.63 & 3066 & 30.40 & 20862 & 61.88 & $<0.001$ \\
\hline 28-365 days & 7925 & 18.09 & 1928 & 19.12 & 5997 & 17.79 & \\
\hline$>365$ days & 11949 & 27.28 & 5092 & 50.49 & 6857 & 20.34 & \\
\hline \multicolumn{8}{|l|}{ Aspirin } \\
\hline$<28$ days & 28319 & 64.65 & 4161 & 41.26 & 24158 & 71.65 & $<0.001$ \\
\hline $28-365$ days & 7385 & 16.86 & 2296 & 22.76 & 5089 & 15.09 & \\
\hline$>365$ days & 8098 & 18.49 & 3629 & 35.98 & 4469 & 13.25 & \\
\hline \multicolumn{8}{|c|}{ Urbanization level } \\
\hline Urban & 30539 & 69.72 & 7208 & 71.47 & 23331 & 69.20 & $<0.001$ \\
\hline Suburban & 8914 & 20.35 & 1920 & 19.04 & 6994 & 20.74 & \\
\hline Rural & 4349 & 9.93 & 958 & 9.50 & 3391 & 10.06 & \\
\hline \multicolumn{8}{|c|}{ Monthly income (NT\$) } \\
\hline 0 & 3464 & 7.91 & 795 & 7.88 & 2669 & 7.92 & $<0.001$ \\
\hline $1-21000$ & 15001 & 34.25 & 3067 & 30.41 & 11934 & 35.40 & \\
\hline $21000-33300$ & 12904 & 29.46 & 3165 & 31.38 & 9739 & 28.89 & \\
\hline$\geq 33301$ & 12433 & 28.38 & 3059 & 30.33 & 9374 & 27.80 & \\
\hline
\end{tabular}

a Comparison between statin use and no statin use.

+CCI: Charlson comorbidity index.

Table 2. All-Cancer Risk in Statin Users and Nonusers in the Study Cohort.

\begin{tabular}{|c|c|c|c|c|c|c|c|c|c|}
\hline \multirow[t]{2}{*}{$\begin{array}{l}\text { Entire cohort } \\
(\mathrm{n}=43,802)\end{array}$} & \multicolumn{4}{|c|}{$\begin{array}{c}\text { Patients not using statins } \\
\text { (Total follow-up: } 194,933.6 \text { person-years) }\end{array}$} & \multicolumn{4}{|c|}{$\begin{array}{l}\text { Patients using statins } \\
\text { (Total follow-up: 80,239.4 person-years) }\end{array}$} & \multirow{2}{*}{$\begin{array}{l}\text { aHR+ } \\
(95 \% \mathrm{CI})\end{array}$} \\
\hline & \multirow{2}{*}{$\begin{array}{l}\text { No. of patients } \\
\text { with any cancer }\end{array}$} & \multicolumn{3}{|c|}{$\begin{array}{l}\text { Incidence rate (per } 10^{5} \text { person-years) } \\
(95 \% \mathrm{CI})\end{array}$} & \multirow{2}{*}{$\begin{array}{l}\text { No. of patients } \\
\text { with any cancer }\end{array}$} & \multicolumn{3}{|c|}{$\begin{array}{l}\text { Incidence rate (per } 10^{5} \text { person-years) } \\
(95 \% \mathrm{CI})\end{array}$} & \\
\hline \multicolumn{8}{|l|}{ Entire cohort } & & \\
\hline & 5279 & 2708.1 & (2635.0, & 2781.2) & 964 & 1201.4 & (1125.6, & 1277.2) & $0.46(0.43,0.50)^{* * *}$ \\
\hline \multicolumn{10}{|c|}{ Age, 40-64 yearsa } \\
\hline & 2172 & 1868.7 & (1790.1, & 1947.3) & 449 & 889.5 & (807.3, & 971.8) & $0.43(0.39,0.48)^{* * *}$ \\
\hline \multicolumn{10}{|c|}{ Age, $65-74$ yearsb } \\
\hline & 1665 & 3732.1 & (3552.8, & 3911.4) & 350 & 1607.1 & (1438.7, & 1775.5) & $0.45(0.40,0.50)^{* * *}$ \\
\hline \multicolumn{10}{|c|}{ Age, $\geq 75$ years $^{c}$} \\
\hline & 1442 & 4229.7 & (4011.4, & $4448.0)$ & 165 & 2066.3 & (1751.1, & 2381.6) & $0.51(0.43,0.60)^{* * *}$ \\
\hline \multicolumn{10}{|l|}{ Female $^{d}$} \\
\hline & 1874 & 2144.4 & (2047.3, & 2241.5) & 423 & 1011.3 & (914.9, & 1107.6) & $0.44(0.40,0.50)^{* * *}$ \\
\hline \multicolumn{10}{|l|}{ Male } \\
\hline & 3405 & 3166.2 & (3059.8, & 3272.5) & 541 & 1408.5 & (1289.8, & 1527.1) & $0.48(0.43,0.52)^{* * *}$ \\
\hline \multicolumn{10}{|c|}{ aTotal follow-up 116228.5 person-year for patients not using statins and 50476.0 for patients using statins. } \\
\hline \multicolumn{10}{|c|}{ bTotal follow-up 44612.9 person-year for patients not using statins and 21778.3 for patients using statins. } \\
\hline \multicolumn{10}{|c|}{ cTotal follow-up 34092.2 person-year for patients not using statins and 7985.1 for patients using statins. } \\
\hline \multicolumn{10}{|c|}{ dTotal follow-up 87389.9 person-year for patients not using statins and 41828.7 for patients using statins. } \\
\hline \multicolumn{10}{|c|}{ eTotal follow-up 107543.7 person-year for patients not using statins and 38410.7 for patients using statins. } \\
\hline \multicolumn{10}{|c|}{ C.I.: confidence interval } \\
\hline HR: adjusted hi & & & & & & & & & \\
\hline
\end{tabular}


Table 3. Incidence Rate and aHRs of the All-Cancer Risk Associated with Statin Use During the Follow-Up Period in COPD Patients.

\begin{tabular}{|c|c|c|c|c|c|c|c|c|}
\hline \multirow{2}{*}{$\begin{array}{l}\text { Variable } \\
\text { Total statin use }\end{array}$} & \multirow[t]{2}{*}{$\begin{array}{l}\text { No. of } \\
\text { patients }\end{array}$} & \multirow[t]{2}{*}{$\begin{array}{l}\text { No. of } \\
\text { person-years }\end{array}$} & \multirow[t]{2}{*}{$\begin{array}{l}\text { No. of patients } \\
\text { with } \\
\text { any cancer }\end{array}$} & \multicolumn{3}{|c|}{$\begin{array}{l}\text { Incidence Rate } \\
\text { (per } 10^{5} \text { person-years) } \\
(95 \% \mathrm{CI})\end{array}$} & \multirow[t]{2}{*}{$\begin{array}{l}\text { aHR } \\
(95 \% \mathrm{CI})\end{array}$} & \multirow[t]{2}{*}{$\begin{array}{l}P \text { for } \\
\text { Trend }\end{array}$} \\
\hline & & & & & & & & \\
\hline Nonuser (<28 cDDDs) & 33716 & 194933.6 & 5279 & 2708.1 & $(2635.0$, & 2781.2) & 1.00 & $<0.001$ \\
\hline User ( $\geq 28$ cDDDs) & 10086 & 80239.4 & 964 & 1201.4 & (1125.6, & 1277.2) & $0.46(0.43,0.50)^{\star * *}$ & \\
\hline 28-90 cDDDs & 2346 & 17095.6 & 294 & 1719.7 & (1523.2, & 1916.3) & $0.65(0.58,0.73)^{* * *}$ & \\
\hline 91-365 cDDDs & 3215 & 24193.1 & 343 & 1417.8 & (1267.7, & $1567.8)$ & $0.54(0.48,0.60)^{\star * *}$ & \\
\hline$>365$ cDDDs & 4525 & 38950.7 & 327 & 839.5 & $(748.5$ & 930.5) & $0.32(0.29,0.36)^{* * *}$ & \\
\hline \multicolumn{9}{|l|}{ Lipophilia statin use $†$} \\
\hline Nonuser (<28 cDDDs) & 35008 & 204288.0 & 5379 & 2633.0 & $(2562.7$ & 2703.4) & 1.00 & $<0.001$ \\
\hline User ( $\geq 28$ cDDDs) & 8794 & 70885.0 & 864 & 1218.9 & (1137.6, & 1300.2) & $0.57(0.53,0.61)^{* * *}$ & \\
\hline 28-90 cDDDs & 2296 & 17069.8 & 270 & 1581.7 & (1393.1, & 1770.4) & $0.67(0.59,0.75)^{* * *}$ & \\
\hline 91-365 cDDDs & 3012 & 23258.7 & 332 & 1427.4 & (1273.9, & 1581.0) & $0.65(0.58,0.73)^{* * *}$ & \\
\hline$>365$ cDDDs & 3486 & 30556.4 & 262 & 857.4 & (753.6, & $961.3)$ & $0.42(0.37,0.48)^{* * *}$ & \\
\hline \multicolumn{9}{|l|}{ Hydrophilia statin use } \\
\hline Nonuser (<28 cDDDs) & 39878 & 242812.7 & 5974 & 2460.3 & (2397.9, & 2522.7) & 1.00 & $<0.001$ \\
\hline User ( $\geq 28$ cDDDs) & 3924 & 32360.4 & 269 & 831.3 & (731.9, & 930.6) & $0.48(0.42,0.55)^{* * *}$ & \\
\hline 28-90 cDDDs & 1122 & 8876.1 & 102 & 1149.2 & (926.1, & 1372.2) & $0.62(0.51,0.75)^{* * *}$ & \\
\hline 91-365 cDDDs & 1531 & 12432.2 & 94 & 756.1 & $(603.2$ & $909.0)$ & $0.45(0.36,0.55)^{* * *}$ & \\
\hline$>365$ cDDDs & 1271 & 11052.0 & 73 & 660.5 & (509.0, & $812.0)$ & $0.40(0.31,0.50)^{* * *}$ & \\
\hline \multicolumn{9}{|l|}{$\begin{array}{l}\text { Individual statin use } \\
(\geq 28 \text { cDDDs }) \ddagger\end{array}$} \\
\hline Simvastatin & 3418 & 28625.0 & 257 & 897.8 & (788.0, & 1007.6) & $0.55(0.49,0.63)^{* * *}$ & \\
\hline Lovastatin & 2109 & 18281.5 & 262 & 1433.1 & (1259.6, & 1606.7) & $0.92(0.81,1.04)$ & \\
\hline Atorvastatin & 5484 & 44678.1 & 484 & 1083.3 & (986.8, & 1179.8) & $0.59(0.54,0.65)^{* * *}$ & \\
\hline Fluvastatin & 1510 & 12855.7 & 151 & 1174.6 & (987.2, & 1361.9) & $0.78(0.66,0.92)^{\star *}$ & \\
\hline Pravastatin & 1501 & 12654.5 & 122 & 964.1 & (793.0, & 1135.2) & $0.66(0.55,0.79)^{* * *}$ & \\
\hline Rosuvastatin & 2741 & 22641.7 & 158 & 697.8 & (589.0, & 806.6) & $0.42(0.36,0.49)^{* * *}$ & \\
\hline
\end{tabular}

Main model was adjusted using propensity scores for age, sex, Charlson comorbidity index, diabetes, hypertension, dyslipidemia, urbanization level, and monthly income. †Lipophilia statins included simvastatin, lovastatin, atorvastatin, and fluvastatin. Hydrophilia statins include pravastatin and rosuvastatin. ‡HRs for individual statins were compared between users ( $\geq 28 \mathrm{cDDDs})$ and nonusers (<28 cDDDs).

Table 4. Sensitivity Analysis of aHRs of Statin Use for Reduction of the All-Cancer Risk.

\begin{tabular}{|c|c|c|c|c|c|}
\hline & \multicolumn{4}{|c|}{$\begin{array}{c}\text { Statin use } \\
\text { aHR }(95 \% \text { CI) }\end{array}$} & \multirow[t]{2}{*}{$P$ for Trend } \\
\hline & $<28$ cDDDs & $28-90$ cDDDs & 91-365 cDDDs & $>365$ cDDDs & \\
\hline Main model $\dagger$ & 1.00 & $0.65(0.58,0.73)^{* * *}$ & $0.54(0.48,0.60)^{* * *}$ & $0.32(0.29,0.36)^{* * *}$ & $<0.001$ \\
\hline \multicolumn{6}{|l|}{ Additional covariates $\ddagger$} \\
\hline Main model + Nonstatin lipid-lowering drugs & 1.00 & $0.66(0.59,0.74)^{* * *}$ & $0.56(0.50,0.62)^{* * *}$ & $0.34(0.30,0.38)^{* * *}$ & $<0.001$ \\
\hline Main model + Metformin & 1.00 & $0.65(0.58,0.73)^{* * *}$ & $0.55(0.49,0.61)^{* * *}$ & $0.34(0.30,0.38)^{* * *}$ & $<0.001$ \\
\hline Main model + ACEI & 1.00 & $0.66(0.59,0.74)^{* * *}$ & $0.58(0.52,0.65)^{* * *}$ & $0.38(0.34,0.42)^{* * *}$ & $<0.001$ \\
\hline Main model + Aspirin & 1.00 & $0.66(0.59,0.74)^{* * *}$ & $0.57(0.51,0.63)^{* * *}$ & $0.35(0.32,0.40)^{* * *}$ & $<0.001$ \\
\hline \multicolumn{6}{|l|}{ Subgroup effects } \\
\hline \multicolumn{6}{|l|}{ Age, years } \\
\hline $40-64$ & 1.00 & $0.65(0.55,0.77)^{* * *}$ & $0.50(0.43,0.59)^{\star * *}$ & $0.29(0.25,0.34)^{* * *}$ & $<0.001$ \\
\hline $65-74$ & 1.00 & $0.64(0.52,0.79)^{* * *}$ & $0.54(0.45,0.64)^{* * *}$ & $0.31(0.26,0.37)^{* * *}$ & $<0.001$ \\
\hline$\geq 75$ & 1.00 & $0.67(0.51,0.87)^{* *}$ & $0.54(0.42,0.71)^{* * *}$ & $0.37(0.28,0.49)^{* * *}$ & $<0.001$ \\
\hline \multicolumn{6}{|l|}{ Sex } \\
\hline Female & 1.00 & $0.61(0.51,0.74)^{\star * *}$ & $0.52(0.44,0.62)^{* * *}$ & $0.33(0.28,0.39)^{* * *}$ & $<0.001$ \\
\hline Male & 1.00 & $0.69(0.59,0.80)^{* * *}$ & $0.56(0.48,0.64)^{* * *}$ & $0.32(0.27,0.37)^{* * *}$ & $<0.001$ \\
\hline \multicolumn{6}{|l|}{$\mathrm{CCI}^{+}$} \\
\hline 0 & 1.00 & $0.63(0.50,0.80)^{* * *}$ & $0.51(0.41,0.64)^{* * *}$ & $0.29(0.23,0.36)^{* * *}$ & $<0.001$ \\
\hline 1 & 1.00 & $0.65(0.53,0.81)^{* * *}$ & $0.56(0.46,0.68)^{* * *}$ & $0.32(0.26,0.39)^{* * *}$ & $<0.001$ \\
\hline 2 & 1.00 & $0.60(0.47,0.77)^{* * *}$ & $0.49(0.38,0.63)^{* * *}$ & $0.31(0.24,0.40)^{* * *}$ & $<0.001$ \\
\hline$\geq 3$ & 1.00 & $0.67(0.53,0.85)^{* *}$ & $0.53(0.42,0.66)^{* * *}$ & $0.32(0.26,0.41)^{* * *}$ & $<0.001$ \\
\hline \multicolumn{6}{|l|}{ Diabetes } \\
\hline No & 1.00 & $0.66(0.58,0.76)^{* * *}$ & $0.53(0.46,0.61)^{* * *}$ & $0.31(0.27,0.36)^{* * *}$ & $<0.001$ \\
\hline Yes & 1.00 & $0.60(0.48,0.76)^{* * *}$ & $0.52(0.43,0.62)^{* * *}$ & $0.32(0.26,0.38)^{* * *}$ & $<0.001$ \\
\hline \multicolumn{6}{|l|}{ Dyslipidemia } \\
\hline No & 1.00 & $0.63(0.54,0.73)^{* * *}$ & $0.52(0.45,0.61)^{* * *}$ & $0.28(0.24,0.33)^{\star * *}$ & $<0.001$ \\
\hline Yes & 1.00 & $0.67(0.55,0.82)^{* * *}$ & $0.54(0.46,0.64)^{* * *}$ & $0.36(0.31,0.43)^{* * *}$ & $<0.001$ \\
\hline \multicolumn{6}{|l|}{ Hypertension } \\
\hline No & 1.00 & $0.73(0.62,0.86)^{* * *}$ & $0.53(0.44,0.63)^{* * *}$ & $0.28(0.23,0.34)^{* * *}$ & $<0.001$ \\
\hline Yes & 1.00 & $0.58(0.49,0.69)^{* * *}$ & $0.52(0.45,0.60)^{* * *}$ & $0.32(0.28,0.37)^{* * *}$ & $<0.001$ \\
\hline
\end{tabular}




\begin{tabular}{|c|c|c|c|c|c|}
\hline & \multicolumn{4}{|c|}{$\begin{array}{c}\text { Statin use } \\
\text { aHR }(95 \% \text { CI })\end{array}$} & \multirow[t]{2}{*}{ P for Trend } \\
\hline & $<28$ cDDDs & $28-90$ cDDDs & 91-365 cDDDs & $>365$ cDDDs & \\
\hline \multicolumn{6}{|c|}{ Nonstatin lipid-lowering drugs } \\
\hline$<28$ days & 1.00 & $0.64(0.56,0.73)^{* * *}$ & $0.55(0.49,0.63)^{* * *}$ & $0.32(0.28,0.37)^{* * *}$ & $<0.001$ \\
\hline 28-365 days & 1.00 & $0.89(0.65,1.20)$ & $0.58(0.44,0.77)^{* * *}$ & $0.36(0.27,0.47)^{* * *}$ & $<0.001$ \\
\hline$>365$ days & 1.00 & $0.62(0.30,1.28)$ & $0.76(0.47,1.23)$ & $0.49(0.32,0.76)^{* *}$ & 0.002 \\
\hline \multicolumn{6}{|l|}{ Metformin } \\
\hline$<28$ days & 1.00 & $0.65(0.56,0.74)^{* * *}$ & $0.55(0.48,0.62)^{* * *}$ & $0.31(0.27,0.36)^{* * *}$ & $<0.001$ \\
\hline $28-365$ days & 1.00 & $0.76(0.54,1.07)$ & $0.39(0.26,0.58)^{* * *}$ & $0.35(0.24,0.52)^{* * *}$ & $<0.001$ \\
\hline$>365$ days & 1.00 & $0.77(0.54,1.09)$ & $0.81(0.64,1.03)$ & $0.45(0.36,0.55)^{* * *}$ & $<0.001$ \\
\hline \multicolumn{6}{|l|}{ ACEI } \\
\hline$<28$ days & 1.00 & $0.65(0.54,0.77)^{* * *}$ & $0.54(0.45,0.65)^{* * *}$ & $0.38(0.30,0.48)^{* * *}$ & $<0.001$ \\
\hline $28-365$ days & 1.00 & $0.75(0.61,0.93)^{*}$ & $0.60(0.48,0.76)^{\star \star *}$ & $0.30(0.22,0.41)^{* * *}$ & $<0.001$ \\
\hline$>365$ days & 1.00 & $0.81(0.64,1.02)$ & $0.81(0.67,0.96)^{*}$ & $0.51(0.43,0.59)^{* * *}$ & $<0.001$ \\
\hline \multicolumn{6}{|l|}{ Aspirin } \\
\hline$<28$ days & 1.00 & $0.63(0.53,0.74)^{* * *}$ & $0.53(0.44,0.62)^{* * *}$ & $0.35(0.29,0.42)^{* * *}$ & $<0.001$ \\
\hline $28-365$ days & 1.00 & $0.69(0.55,0.86)^{* *}$ & $0.65(0.52,0.81)^{* * *}$ & $0.30(0.23,0.40)^{* * *}$ & $<0.001$ \\
\hline$>365$ days & 1.00 & $0.96(0.74,1.25)$ & $0.78(0.63,0.96)^{*}$ & $0.51(0.43,0.61)^{* * *}$ & $<0.001$ \\
\hline $\begin{array}{l}{ }^{*} \mathrm{p}<0.05{ }^{* *} \mathrm{p} \\
\text { aHR: adjusted } \\
+ \text { CCI: Charlso }\end{array}$ & & & & & \\
\hline
\end{tabular}

\section{Discussion}

Recently, interest in the function of systemic inflammation in COPD has been increasing.[27-31] Epidemiological studies have shown that elevated levels of systemic inflammatory markers, particularly C-reactive protein (CRP), interleukin 6, and fibrinogen, predict poor outcomes in COPD, including accelerated lung function loss, stronger infective exacerbation propensity, and higher mortality.[32-34] The prevalence of smoking is considerably high among COPD patients: 54\%-77\% among mild COPD patients and 38\%-51\% among severe COPD patients.[35-38] A 25-year follow-up study of a general population in the Danish Death Register revealed that $92 \%$ of the COPD patients have a current or past history of smoking.[39] Smoking reduces the serum high-density lipoprotein (HDL)-cholesterol levels and impairs HDL function by reducing its antioxidant and anti-inflammatory capacity and impeding the cellular cholesterol efflux.[40, 41] Statins reduce CRP levels, independent of their effects on lipids,[42, 43] thus potentially preventing cancers among COPD patients with systemic inflammation and lipid disorder.

Cancers are the leading causes of death in Taiwan. [44] Age is a major risk factor for sporadic cancer. In this study, the strongest chemopreventive effect against all cancers was observed in COPD patients aged $40-75$ years (Table 2). Thus, in Taiwan, statins may have favorable chemoprevention effects in COPD patients at higher all-cancer risk owing to their age. After sex stratification, the aHRs in statin users were lower than statin nonusers (women: $\mathrm{aHR}=$ $0.55,95 \%$ CI: 0.42 to 0.72 ; men: $\mathrm{aHR}=0.44,95 \% \mathrm{CI}$ :
0.40 to 0.50$)$. A similar effect of reduction in the all-cancer risk was observed among COPD patients, regardless of their sex.

In this study, statins reduced the all-cancer risk in COPD patients with dose-dependently regardless of lipophilia or hydrophilia statin use. This is the first article to estimate the dose-dependent chemopreventive effect of statins against all cancers in COPD patients. Hydrophilia statins $(\mathrm{aHR}=0.45,95 \%$ CI: 0.36 to 0.55 ) appeared to have a stronger potential anticancer effect because their moderate dose (91-365 cDDDs) could more sufficiently reduce cancer incidence compared with lipophilia statins $(\mathrm{aHR}=$ $0.65,95 \%$ CI: 0.58 to 0.73 ). On estimating the chemopreventive effects of individual statins against all cancers, we observed the following: the aHRs for the all-cancer risk among patients using rosuvastatin, simvastatin, atorvastatin, pravastatin, and fluvastatin differed (aHRs $=0.42,0.55,0.59,0.66$, and 0.78, respectively). Individual statins reduced the all-cancer risk at varying efficacies among COPD patients. The anticancer efficacies of different statins may be compatible with their lipid-lowering ability. In our study, rosuvastatin had the most predominant chemoprevention effect against all cancers in COPD patients. Rosuvastatin, atorvastatin, and simvastatin also caused the highest percent change in LDL-cholesterol levels. Rosuvastatin is slightly more potent than is atorvastatin, and both these agents are significantly more potent than simvastatin, lovastatin, pravastatin, and fluvastatin.[45, 46] At maximal prescribed doses, the reduction in LDL levels is larger with rosuvastatin and atorvastatin than with the other available statins.[47] This study is the first to estimate the anticancer efficacies of statins and compatible 
with the potency of for lowering LDL levels. These outcomes can indicate a statin ideal for further clinical studies

A higher proportion of statins nonuser used nonstatin lipid-lowering drugs, metformin, ACEI, and aspirin for <28 cDDDs; however, most statin users used these for $\geq 28 \mathrm{cDDDs}$. If moderate to high cDDDs (28-365 or $>365$ cDDDs) of aspirin, metformin, and ACEI are used, the chemopreventive effect of statins against the all cancer risk will be masked (Table 4). If statin dose is increased to $>365$ cDDDs, the aHRs of statins for reducing all-cancer risk in COPD patients were significant in our sensitivity analysis. These outcomes might explain the independent chemopreventive effect of aspirin, metformin, ACEI, nonstatin lipid-lowering drugs, and statins.[48, 49] However, the anticancer effects of statins, associated with anti-inflammatory, antioxidant, antiplatelet, and lipid modification effects, cannot be replaced by aspirin, metformin, ACEI, and nonstatin lipid-lowering drug use.[14-16] This is also the first study to propose that statins exerts dose-response and chemopreventive effects against all cancers in COPD patients.

However, this study has potential limitations. The bias of additional risk factors associated with COPD and all cancers, including a personal or family history of sporadic cancers, obesity, alcohol use, physical activity, and smoking, could not be eliminated. A large-scale randomized trial with a suitable regimen in well-selected patients comparing standard approaches is required for obtaining this crucial information. However, methodological concerns may obscure the precise relationship between these factors and cancer risk. In our study, we used PSs to match age, sex, the CCI, diabetes, hypertension, dyslipidemia, urbanization levels, and monthly income. The urbanization level and monthly income are invalidated alternatives for lifestyle factors and the environmental level. Moreover, the diagnoses of all cancers and other comorbidities were completely dependent on the ICD-9-CM codes. Nevertheless, the NHI Administration randomly reviews medical records and interviews patients to validate diagnoses, and hospitals with outlier diagnoses and practices may be audited and subsequently penalized heavily if malpractice and discrepancies are discovered. Another limitation of this study is that information on several unmeasured confounders, such as body mass index, smoking, alcohol use, and other over-the-counter drug use-which are associated with cancers-is unavailable in the NHIRD. However, considering the magnitude and significance of the observed effects, it is unlikely that these limitations have compromised the results. Finally, because our study is not a prospective randomized blinded study, a cause-effect relationship could not be established. The findings of this study suggest that statins dose-dependently exert a significant chemopreventive effect against all cancers in COPD patients. Additional randomized studies are required to verify these findings.

\section{Conclusions}

Statins dose-dependently exert a significant chemopreventive effect against all cancers in COPD patients; in particular, rosuvastatin has the strongest chemopreventive effect.

\section{Competing Interests}

The authors have declared that no competing interest exists.

\section{References}

1. Gershon AS, Warner L, Cascagnette P, Victor JC, To T. Lifetime risk of developing chronic obstructive pulmonary disease: a longitudinal population study. Lancet. 2011; 378: 991-6.

2. Sin DD, Paul Man SF. Cooling the fire within: inhaled corticosteroids and cardiovascular mortality in COPD. Chest. 2006; 130: 629-31.

3. Thompson PA, Khatami M, Baglole CI, Sun J, Harris SA, Moon EY, et al. Environmental immune disruptors, inflammation and cancer risk. Carcinogenesis. 2015; 36 Suppl 1: S232-53.

4. Bozinovski S, Vlahos R, Anthony D, McQualter J, Anderson G, Irving L, et al. COPD and squamous cell lung cancer: aberrant inflammation and immunity is the common link. British journal of pharmacology. 2016; 173: 635-48.

5. Hole DJ, Watt GC, Davey-Smith G, Hart CL, Gillis CR, Hawthorne VM. Impaired lung function and mortality risk in men and women: findings from the Renfrew and Paisley prospective population study. Bmj. 1996; 313: 711-5; discussion 5-6.

6. Kuller LH, Ockene J, Meilahn E, Svendsen KH. Relation of forced expiratory volume in one second (FEV1) to lung cancer mortality in the Multiple Risk Factor Intervention Trial (MRFIT). American journal of epidemiology. 1990; 132: 265-74.

7. Shen Y, Yang T, Guo S, Li X, Chen L, Wang T, et al. Increased serum ox-LDL levels correlated with lung function, inflammation, and oxidative stress in COPD. Mediators of inflammation. 2013; 2013: 972347.

8. Jung YS, Ryu S, Chang Y, Yun KE, Park JH, Kim HJ, et al. Associations Between Parameters of Glucose and Lipid Metabolism and Risk of Colorectal Neoplasm. Digestive diseases and sciences. 2015; 60: 2996-3004.

9. McDonnell DP, Park S, Goulet MT, Jasper J, Wardell SE, Chang CY, et al. Obesity, cholesterol metabolism, and breast cancer pathogenesis. Cancer research. 2014; 74: 4976-82.

10. Chavarro JE, Kenfield SA, Stampfer MJ, Loda M, Campos H, Sesso HD, et al. Blood levels of saturated and monounsaturated fatty acids as markers of de novo lipogenesis and risk of prostate cancer. American journal of epidemiology. 2013; 178: 1246-55.

11. Sevenoaks MJ, Stockley RA. Chronic Obstructive Pulmonary Disease, inflammation and co-morbidity--a common inflammatory phenotype? Respiratory research. 2006; 7: 70.

12. MacNee W. Pulmonary and systemic oxidant/antioxidant imbalance in chronic obstructive pulmonary disease. Proceedings of the American Thoracic Society. 2005; 2: 50-60.

13. Matakidou A, Eisen T, Houlston RS. Systematic review of the relationship between family history and lung cancer risk. British journal of cancer. 2005; 93: 825-33.

14. Pignatelli P, Carnevale R, Pastori D, Cangemi R, Napoleone L, Bartimoccia S, et al. Immediate antioxidant and antiplatelet effect of atorvastatin via inhibition of Nox2 Circulation. 2012; 126: 92-103.

15. Ridker PM, Rifai N, Pfeffer MA, Sacks FM, Moye LA, Goldman S, et al. Inflammation, pravastatin, and the risk of coronary events after myocardial infarction in patients with average cholesterol levels. Cholesterol and Recurrent Events (CARE) Investigators. Circulation. 1998; 98: 839-44.

16. Horne BD, Muhlestein JB, Carlquist JF, Bair TL, Madsen TE, Hart NI, et al. Statin therapy, lipid levels, C-reactive protein and the survival of patients with angiographically severe coronary artery disease. Journal of the American College of Cardiology. 2000; 36: 1774-80.

17. Cao C, Wu Y, Xu Z, Lv D, Zhang C, Lai T, et al. The effect of statins on chronic obstructive pulmonary disease exacerbation and mortality: a systematic 
review and meta-analysis of observational research. Scientific reports. 2015; 5: 16461.

18. Hussein O, Schlezinger S, Rosenblat M, Keidar S, Aviram M. Reduced susceptibility of low density lipoprotein (LDL) to lipid peroxidation after fluvastatin therapy is associated with the hypocholesterolemic effect of the drug and its binding to the LDL. Atherosclerosis. 1997; 128: 11-8.

19. Girona J, La Ville AE, Sola R, Plana N, Masana L. Simvastatin decreases aldehyde production derived from lipoprotein oxidation. The American journal of cardiology. 1999; 83: 846-51.

20. Baigent C, Blackwell L, Emberson J, Holland LE, Reith C, Bhala N, et al. Efficacy and safety of more intensive lowering of LDL cholesterol: a meta-analysis of data from 170,000 participants in 26 randomised trials. Lancet. 2010; 376: 1670-81.

21. Dale KM, Coleman CI, Henyan NN, Kluger J, White CM. Statins and cancer risk: a meta-analysis. JAMA : the journal of the American Medical Association. 2006; 295: 74-80.

22. Chen CI, Kuan CF, Fang YA, Liu SH, Liu JC, Wu LL, et al. Cancer risk in HBV patients with statin and metformin use: a population-based cohort study. Medicine (Baltimore). 2015; 94: e462.

23. Shao JY, Lee FP, Chang CL, Wu SY. Statin-Based Palliative Therapy for Hepatocellular Carcinoma. Medicine (Baltimore). 2015; 94: e1801.

24. Singh S, Singh PP. Statins for prevention of hepatocellular cancer: one step closer? Hepatology. 2014; 59: 724-6.

25. D'Agostino RB, Jr. Propensity score methods for bias reduction in the comparison of a treatment to a non-randomized control group. Statistics in medicine. 1998; 17: 2265-81.

26. Schneeweiss S. Sensitivity analysis and external adjustment for unmeasured confounders in epidemiologic database studies of therapeutics. Pharmacoepidemiology and drug safety. 2006; 15: 291-303.

27. Young RP, Hopkins R, Eaton TE. Pharmacological actions of statins: potential utility in COPD. European respiratory review : an official journal of the European Respiratory Society. 2009; 18: 222-32.

28. Sin DD, Man SF. Why are patients with chronic obstructive pulmonary disease at increased risk of cardiovascular diseases? The potential role of systemic inflammation in chronic obstructive pulmonary disease. Circulation. 2003; 107: 1514-9.

29. Barnes PJ, Celli BR. Systemic manifestations and comorbidities of COPD. The European respiratory journal. 2009; 33: 1165-85.

30. Barr RG. The epidemiology of vascular dysfunction relating to chronic obstructive pulmonary disease and emphysema. Proceedings of the American Thoracic Society. 2011; 8: 522-7.

31. Fabbri LM, Beghe B, Agusti A. Cardiovascular mechanisms of death in severe COPD exacerbation: time to think and act beyond guidelines. Thorax. 2011; 66: 745-7.

32. Sin DD, Man SF. Interleukin-6: a red herring or a real catch in COPD? Chest. 2008; 133: 4-6.

33. Cazzola M, Page CP, Calzetta L, Matera MG. Emerging anti-inflammatory strategies for COPD. The European respiratory journal. 2012; 40: 724-41.

34. Ahmad T, Mabalirajan U, Sharma A, Aich J, Makhija L, Ghosh B, et al. Simvastatin improves epithelial dysfunction and airway hyperresponsiveness: from asymmetric dimethyl-arginine to asthma. American journal of respiratory cell and molecular biology. 2011; 44: 531-9.

35. Wedzicha JA, Calverley PM, Seemungal TA, Hagan G, Ansari Z, Stockley RA. The prevention of chronic obstructive pulmonary disease exacerbations by salmeterol/fluticasone propionate or tiotropium bromide. American journal of respiratory and critical care medicine. 2008; 177: 19-26.

36. Vestbo J, Sorensen T, Lange P, Brix A, Torre P, Viskum K. Long-term effect of inhaled budesonide in mild and moderate chronic obstructive pulmonary disease: a randomised controlled trial. Lancet. 1999; 353: 1819-23.

37. Watson L, Vonk JM, Lofdahl CG, Pride NB, Pauwels RA, Laitinen LA, et al. Predictors of lung function and its decline in mild to moderate COPD in association with gender: results from the Euroscop study. Respiratory medicine. 2006; 100: 746-53.

38. Calverley PM, Anderson JA, Celli B, Ferguson GT, Jenkins C, Jones PW, et al. Salmeterol and fluticasone propionate and survival in chronic obstructive pulmonary disease. The New England journal of medicine. 2007; 356: 775-89.

39. Lokke A, Lange P, Scharling H, Fabricius P, Vestbo J. Developing COPD: a 25 year follow up study of the general population. Thorax. 2006; 61: 935-9.

40. Rosenson RS, Brewer HB, Jr., Ansell BJ, Barter P, Chapman MJ, Heinecke JW, et al. Dysfunctional HDL and atherosclerotic cardiovascular disease. Nature reviews Cardiology. 2016; 13: 48-60.

41. Lipids and lipoproteins in symptomatic coronary heart disease. Distribution, intercorrelations, and significance for risk classification in 6,700 men and 1,500 women. The Bezafibrate Infarction Prevention (BIP) Study Group, Israel. Circulation. 1992; 86: 839-48.

42. Vernaglione L, Cristofano C, Muscogiuri P, Chimienti S. Does atorvastatin influence serum C-reactive protein levels in patients on long-term hemodialysis? American journal of kidney diseases : the official journal of the National Kidney Foundation. 2004; 43: 471-8.

43. Kumar S, Raftery M, Yaqoob M, Fan SL. Anti-inflammatory effects of 3-hydroxy-3-methylglutaryl coenzyme a reductase inhibitors (statins) in peritoneal dialysis patients. Peritoneal dialysis international : journal of the International Society for Peritoneal Dialysis. 2007; 27: 283-7.
44. Health Promotion Administration MoHaW. Taiwan Cancer Registry report, 2011 edition. Health Promotion Administration, Ministry of Health and Welfare; 2011.

45. Rosenson RS. Rosuvastatin: a new inhibitor of HMG-coA reductase for the treatment of dyslipidemia. Expert review of cardiovascular therapy. 2003; 1 : 495-505.

46. Jones PH, Davidson MH, Stein EA, Bays HE, McKenney JM, Miller E, et al. Comparison of the efficacy and safety of rosuvastatin versus atorvastatin, simvastatin, and pravastatin across doses (STELLAR* Trial). The American journal of cardiology. 2003; 92: 152-60.

47. Brown AS, Bakker-Arkema RG, Yellen L, Henley RW, Jr., Guthrie R, Campbell $\mathrm{CF}$, et al. Treating patients with documented atherosclerosis to National Cholesterol Education Program-recommended low-density-lipoprotein cholesterol goals with atorvastatin, fluvastatin, lovastatin and simvastatin. Journal of the American College of Cardiology. 1998; 32: 665-72.

48. Gronich N, Rennert G. Beyond aspirin-cancer prevention with statins, metformin and bisphosphonates. Nature reviews Clinical oncology. 2013; 10: 625-42.

49. Janne PA, Mayer RJ. Chemoprevention of colorectal cancer. The New England journal of medicine. 2000; 342: 1960-8. 